\title{
Développement d'une source EUV plasma laser pour la micro-lithographie
}

\author{
M. Segers, M. Bougeard, E. Caprin, T. Ceccotti, F. Chichmanian, D. Descamps, \\ P. Haltebourg, J.-F. Hergott, S. Hulin, D. Normand, M. Schmidt et O. Sublemontier
}

Groupe d'Applications des Plasmas, CEA-DSM/DRECAM/SPAM, CE-Saclay, bâtiment 522, 91191 Gif-sur-Yvette cedex, France

\begin{abstract}
Résumé. Le Groupe des Applications Plasma (GAP) du CEA à Saclay participe au projet national PREUVE du Réseau Micro- et Nano-Technologies. Ce projet a été lancé fin 1999 pour réunir et développer les compétences en France sur la lithographie dans l'extrême ultraviolet (LEUV). Au sein de PREUVE, notre objectif a été le développement d'une source plasma laser dans l'EUV autour de $13 \mathrm{~nm}$ afin de contribuer à la réalisation d'un premier banc d'essai pour la lithographie (BEL) en Europe. Afin de réaliser cette source, nous utilisons un plasma émetteur qui est produit par l'interaction d'un laser de type Nd:YAG sur un jet de gouttelettes de xénon. A la fin du projet PREUVE, cette source satisfait les principales spécifications et répond en particulier aux besoins en flux de photons EUV pour réaliser des tests d'insolation EUV avec le banc d'essai. Suite à ces résultats prometteurs, nous démarrons actuellement un projet industriel EXULITE avec nos partenaires du CEA, d'Alcatel et de Thalès sur le développement d'une source EUV de puissance pour des machines de lithographie de production. Ce projet se terminera en 2005.
\end{abstract}

\section{INTRODUCTION}

La lithographie EUV prendra le relais de la lithographie optique en 2007 pour la microélectronique avancée afin de réaliser des circuits intégrés de dimensions critiques inférieures ou égales à $0.05 \mu \mathrm{m}$. Cette nouvelle génération de lithographie devrait permettre de continuer à respecter la loi de Moore [1], qui stipule que la puissance des circuits imprimés double approximativement tous les dix-huit mois. Le BEL sera la première machine EUV en Europe permettant de faire les premiers tests d'insolation de résines. Il est actuellement monté en salle blanche au CEA-LETI à Grenoble. Un des points critiques de la réalisation d'une machine pour la lithographie EUV est le développement d'une source EUV à la fois intense et propre, i.e. sans débris, pour préserver la qualité des optiques de collection sur deux années de fonctionnement. Au sein de PREUVE, notre groupe a développé une source plasma laser émettant dans l'EUV appelée EUV Lithography Source Apparatus (ELSA). Dans le but d'optimiser cette source ELSA, nous avons mis en place un ensemble de diagnostics pour sa caractérisation complète. Cette caractérisation nous est aujourd'hui fort utile pour le développement d'une source de puissance pour les machines industrielles de lithographie EUV dans le cadre du projet EXULITE. Ce projet fait parti du programme européen MEDEA+, en collaboration avec Alcatel, Thalès Laser et le CEA-SPAL.

\section{REALISATION D'UNE SOURCE EUV}

\subsection{Choix de la source}

Dans le cadre de PREUVE, trois types de sources ont été développés en parallèle dans le but de pousser les recherches dans les voies les plus prometteuses et de trouver ainsi la source la plus adaptée au BEL. Une source à décharges capillaire (CAPELLA) à été mise au point par le CNRS-GREMI à Orléans. Deux sources plasma-laser ont été développées, l'une par le CEA-DAM utilisant pour cible un film métallique d'épaisseur micrométrique qui est irradié en face arrière, et l'autre développée par notre groupe le GAP du CEA-DSM-SPAM à Saclay, qui a pour cible un jet dense et directif formé de gouttelettes de xénon (ELSA fig. 1). 


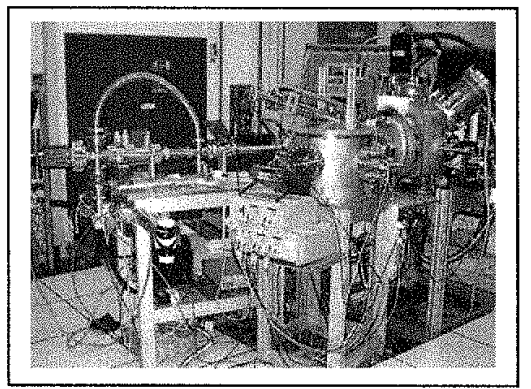

Figure 1. Montage expérimental d'ELSA et des différents diagnostics EUV.

\subsection{Paramètres du jet}

En considérant les contraintes de production des futurs steppers EUV ( 80 plaquettes $/ h$ ) la récurrence des sources EUV sera de l'ordre de $5 \mathrm{kHz}$ ou plus. De ce point de vue, les jets représentent la meilleure cible pour une source plasma laser car les vitesses d'écoulement sont de l'ordre de plusieurs dizaines de mètres par seconde, assurant ainsi le renouvellement rapide de la matière émettrice dans la zone d'interaction. Cela nous permet d'apporter une réponse aux contraintes de cadence. Cependant, un inconvénient important des jets est la faible densité par rapport à une cible solide, ce qui réduit drastiquement le couplage avec le laser surtout quand on est obligé pour des raisons techniques d'attaquer le jet en aval à plusieurs centimètres de la buse de l'injecteur. En effet, différents types de jets (Fig. 2) ont été développés pour cette application spécifique, offrant chacun leurs avantages et leurs défauts. Ainsi dans le cas d'un jet d'agrégats, il y a une forte probabilité que le rayonnement EUV produit par le plasma soit réabsorbé par l'enveloppe gazeuse. Dans le cas d'un micro-jet liquide, la zone d'interaction est plus étroite, mais rend la cible instable par la production d'ondes de chocs dans le jet due à l'interaction avec le laser.

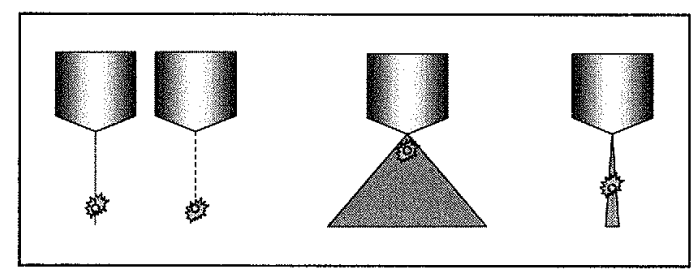

Figure 2. schémas des différents types de jet.

Dans le cadre d'ELSA, nous avons élaboré un jet qui concilie les qualités des autres, tout en réduisant leurs défauts, en réalisant un jet de gouttelettes dense et confiné $\left(D_{\mathrm{jet}}=10^{20} \mathrm{~atm} / \mathrm{cm}^{3}, \alpha<5^{\circ}\right)$. Une étude sur la dimension des gouttelettes et la qualité du couplage a été menée pour différentes géométries de buses en régime pulsé, et dans différentes conditions thermodynamiques. Cela nous a permis de déterminer les conditions optimales de génération, et de déposer un brevet sur la réalisation d'une telle cible (Fig. 3a).

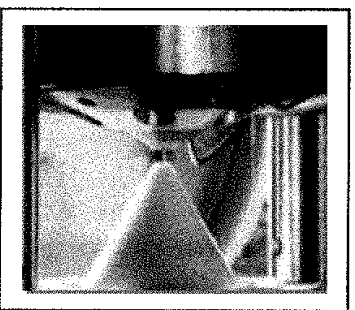

a)

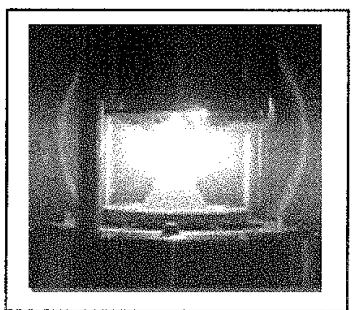

b)

Figure 3. Image de la source ELSA sans (a) et avec laser (b). 


\subsection{Paramètres du laser}

La technologie laser Nd:YAG représente la solution la moins onéreuse pour des lasers de pompe fonctionnant à haute cadence. Notre source est optimisée pour fonctionner avec cette technologie laser pour des impulsions de plusieurs nano-secondes (Fig. 3b).

Notre source est caractérisée à l'aide d'un ensemble de diagnostics EUV calibré, développé dans notre laboratoire, semblable au «flying circus » monté par ASML et Philips [2]. Ainsi, nous sommes en mesure de déterminer précisément le flux de photon autour de 13,5nm à l'aide d'un reflectomètre calibré par le PTB à Berlin sur BESSY 2, de caractériser spectralement le rayonnement EUV produit (Fig. 4a) avec un spectromètre à réseau en transmission [3], et d'imager la source avec une résolution inférieure à $50 \mu \mathrm{m}$. Concernant la propreté de la source nous avons développé un spectromètre à temps de vol afin de caractériser les débris émis par la source [4]. Cet ensemble de diagnostics nous permet d'étudier l'influence de différents paramètres, comme le profil temporel du laser ou la forme du jet, sur le rendement et la propreté de notre source.
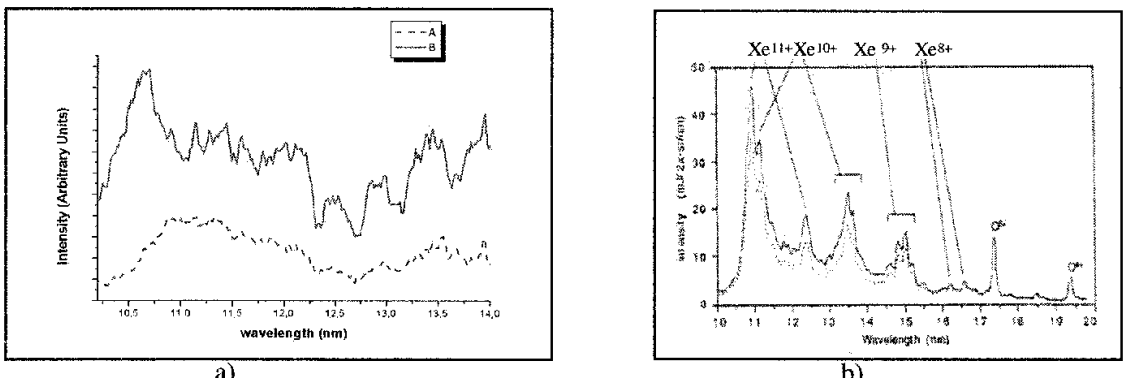

Figure 4. (a) Spectres EUV obtenus pour un jet d'agrégats (trait pointillé A) et pour notre jet (trait plein B), et (b) spectre d'une source à décharge capillaire [5].

Parallèlement, un programme de simulations en collaboration avec différents laboratoires ${ }^{1}$ a été conduit pour étudier l'influence de différents paramètres temporels du laser sur la qualité du couplage avec la cible. Dans le but de valider les résultats fournis par des simulations nous avons développé un système optique permettant de modifier la durée et le profil temporel de l'impulsion. Une étude est en cours afin d'observer l'évolution de l'efficacité de conversion en fonction de la durée de l'impulsion laser de pompe. Ceci est fait à éclairement constant, afin de conserver la même température électronique $\mathrm{T}_{\mathrm{e}} \mathrm{du}$ plasma donnant la population ionique émettrice dominante. Typiquement pour un éclairement laser de $5 \times 10^{11} \mathrm{~W} \mathrm{~cm}^{-2}$ on obtient une température électronique d'environ $40-60 \mathrm{eV}$. A cette température la population ionique majoritaire est centrée autour de l'ion $\mathrm{Xe}^{10+}$ qui contribue principalement à la bande d'émission autour de $13,5 \mathrm{~nm}[6]$.

\section{OPTIMISATION DE LA SOURCE}

\subsection{Performances de la source}

Nous disposons actuellement d'un laser commercial Nd:YAG qui fonctionne à un taux de répétition de $50 \mathrm{~Hz}$ et délivre des impulsions d'une durée de $8 \mathrm{~ns}$ et d'une énergie de $1,2 \mathrm{~J}$ à $\lambda_{1}=1,064 \mu \mathrm{m}$. Les spectres que nous avons obtenus (Fig. 4a) avec notre source ELSA sont très proches de ceux d'une source à décharge capillaire [7]. Dans ces conditions, l'efficacité de conversion (CE) laser-EUV atteint actuellement $0,57 \%$ à $13,5 \mathrm{~nm}$ dans $2 \pi \mathrm{sr}$ d'angle solide et $2 \%$ de bande passante $(0,27 \mathrm{~nm})$. Cependant, pour le BEL l'angle de collection est seulement de $0,2 \mathrm{sr}$. Avec une puissance laser de $40 \mathrm{~W}$, on obtient ainsi une puissance EUV pouvant être collectée de l'ordre de $7 \mathrm{~mW}$. Pour les besoins du BEL, ces performances s'avèrent largement suffisantes.

T. Blensky (CEA-DSM), J.-C. Gauthier (LULD), et O. Peyrusse (CEA-DAM). 
Pour l'industrialisation de notre source le cahier des charges est beaucoup plus contraignant que celui du BEL, notamment en ce qui concerne le flux EUV requis ainsi que la propreté de la source afin d'assurer une durée de vie suffisante de l'optique de collection EUV. Il est demandé de produire au minimum $25 \mathrm{~W}$ d'EUV utiles à une cadence de $10 \mathrm{kHz}$, ce qui correspond à $0.8 \mathrm{~mJ} / \mathrm{tir} / \mathrm{sr}$ collectés. Compte tenu de notre $\mathrm{CE}$ actuel cela nécessite de réaliser une source laser capable de délivrer plus de 5 $\mathrm{kW}$. L'augmentation de la puissance se manifestera avant tout par l'apparition de contraintes thermiques massives qui auront pour conséquence une détérioration prématurée du système (injecteur, optique EUV). Des études seront donc à mener sur ce problème pour allonger la durée de vie du système.

\subsection{Développement du prototype}

Le projet EXULITE, dont le maitre d'œuvre est Alcatel Vacuum Technology France (AVTF), a débuté en 2001 dans le cadre de consortium européen MEDEA+ en collaboration avec Thalès laser, et deux laboratoires CEA (DSM/SPAM et DEN/SPAL). Nous avons en charge le développement d'une source laser-plasma pour trouver une solution industrielle fonctionnant à haute cadence $(10 \mathrm{kHz})$. En ce qui concerne la cible, nous devons passer à un jet continu de gouttelettes de xénon pour assurer le renouvellement de la matière dans le foyer du laser à la cadence souhaitée. Le dispositif laser retenue est composé de plusieurs modules laser Nd:YAG nécessitant ensuite un multiplexage des faisceaux sur la cible (Fig. 5). Durant PREUVE Thalès Laser a travaillé sur la réalisation de modules laser, pompé par diode continue, fonctionnant à $1,064 \mu \mathrm{m}$ et délivrant $300 \mathrm{~W}$ à $10 \mathrm{kHz}$. D'autres études sont à mener pour augmenter la puissance de ces modules et réduire leur nombre dans notre dispositif. Cette architecture a plusieurs avantages décisifs, notamment un faible coût de développement, la simplicité, une grande fiabilité de chaque module, et enfin une redondance importante du système final.

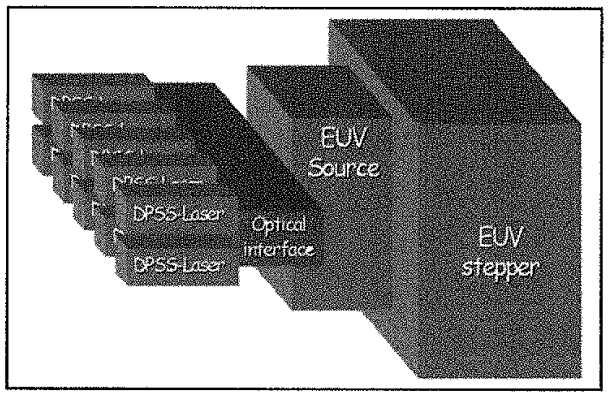

Figure 5. Schéma du montage modulaire des lasers de pompe actuellement développé dans le cadre d'EXULITE

\section{CONCLUSION}

Notre source ELSA est désormais opérationnelle et fonctionne de façon satisfaisante pour les besoins définis pour le BEL. En vue d'une industrialisation dans le cadre du projet EXULITE, les compétences acquises lors du projet PREUVE nous permettent d'envisager le développement d'une source EUV de forte puissance fonctionnant à $10 \mathrm{kHz}$. Le prototype de cette source devrait être réalisé d'ici 2005.

\section{Références}

[1] G.E. Moore, Electronics 38, 114 (1965).

[2] M. Schmidt et al., International SEMATECH EUV Workshop, San Francisco, 19-21 October 2000.

[3] T. Wilhein et al., Rev. Sci. Instr. 70, 1694, (1999).

[4] O. Sublemontier et al., en préparation.

[5] M. A. Klosner and W. T. Silfvast, Opt. Lett. 23, 1609 (1998).

[6] F.Gilleron et al., en preparation

[7] M. A. Klosner and W.T. Silfvast, J. Opt. Soc. Am. B 17, 1279 (2000). 